labour. The 3-rd group consisted of 2078 workers, hard manual labour without influence of bone-seeking toxic factors (metalworker, painters, moulders etc.). The 4-th group - 3240 workers and employees of auxiliary departments not subjected to the influence of unfavourable industrial factors (engineer, command, economists etc.). The observed were divided into four age groups and three working experience groups.

Results In the observed population the prevalence of SPS was 9,6 (95\% CI 8,9-10,7), among male - 8,6 (7,8-9,4), among female - 1,9 $(10,5-13,5)$. The highest rates of SPS were aged 40 to 49 years - $11,0(9,6-12,4)$ and older than 50 years - 11,9 $(10,5-13,3)$. The highest prevalence index of SPS were registered in the B first group - 32, 9\% (workers exposed to toxic effects of fluoride and physical strain), the lowest - in the 3-rd group - 6,9\% (without the impact of toxic action). Rate of shoulder pain prevalence is authentically higher among the workers of hard manual labour and under the toxic influence of fluoride. The prevalence of SP in the 3rd group was similar to the index of the 4th group.

Conclusions The highest RR of SPS progress was observed in the 1 st group in the relation to 4 th $(5,6)$ and 3 rd groups $(5,2)$, at the same time etiological fraction (EF) was $77.5 \%$ and $78,7 \%$, it indicates very high influence of labour conditions on prevalence of SPS. Influence without the manual labour index of RR and EF is much higher in the 2nd group, than in 3rd and 4th groups.

It's worth noting that the highest prevalence of SPS was found among patients with broncho-pulmonary system diseases $24,8(20,1-29,4)$, it is higher than among patients with neck pain - 23,4 (21,5-25,3). Among the analysed "nonmanufactoring" data co-morbid pathology of the respiratory system and neck pain increase the risk of the shoulder pain syndrome development.

The relative risk of SPS was higher among patients with neck pain $(7,0)$ than with comorbidity of broncho-pulmonary system $(2,6)$.

\section{AN OLD TRADE WITH AN UNANSWERED QUESTION: DOES ARC-WELDING FUME EXPOSURE INCREASE THE RISKS OF OBSTRUCTIVE PULMONARY DISEASES? FIRST FINDINGS FROM THE WELSHIP CROSS-SECTIONAL STUDY}

${ }^{1}$ Andrea Marongiu, ${ }^{2}$ Omer Hasan, ${ }^{2}$ Anila Ali, ${ }^{2}$ Sharoon Bakhsh, ${ }^{2}$ Bobby George, ${ }^{2}$ Nabeel Irfan, ${ }^{1}$ Cristina Canova, ${ }^{1}$ Susie Schofield, ${ }^{1} J o a n a$ Szram, ${ }^{1}$ Cosetta Minelli, ${ }^{1}$ Paul Cullinan. 'Imperial College London, London, UK; ${ }^{2}$ Drydocks World Dubai, Dubai, United Arab Emirates

\subsection{6/oemed-2014-102362.362}

Objectives Increasingly, global manufacturing is shifting to emerging economies and with it the use of arc-welding for applications in different industries. The chronic respiratory adverse effects resulting from exposure to gases and ultrafine metal particles in welding fume are incompletely understood.

We aimed to measure the prevalence of arc-welding related pulmonary obstructive outcomes by analysing data collected in a shipyard in the Middle East.

Method Between January and December 2013, through crosssectional survey, we collected spirometry data and behavioural, occupational and respiratory symptoms information from a random sample of male shipyard workers; 397 were exposed to welding fume and 127 were non-exposed. The sample was selected from a total population of about 8000 employees, by frequency matching for ethnicity and age relatively to full-time welders ('highly' exposed).

Results Of the 580 workers invited, 26 subsequently left their job; of the remainder, $95 \%(524)$ agreed to participate. The participants, from the Indian subcontinent $(90 \%)$ or Philippines (10\%), had a median age of 38 years. Ever smoking was reported by $37 \%$, with full-time welders reporting the lowest proportion of current smoking, 18\%(24/131).

Overall, $13 \%$ reported respiratory symptoms with a higher prevalence in the winter months. Post-bronchodilator spirometry data were available for $91 \%$ of workers. Mean values for FEV1 and FVC were $2.87 \mathrm{~L}$ and $3.48 \mathrm{~L}$, with no statistically significant differences across exposure groups (p-values: 0.71 and $0.48)$.

Conclusions These preliminary results need to be explored further in relation to smoking, past and current occupational exposure. This population, it is hoped, will form the basis for a longitudinal study.

\section{PUBMED SEARCH STRINGS FOR THE STUDY OF PUTATIVE ENVIRONMENTAL DETERMINANTS OF DISEASE}

${ }^{1}$ Stefano Mattioli, ${ }^{2}$ Davide Gori, ${ }^{2}$ Valentina Di Gregori, ${ }^{1}$ Andrea Farioli, ${ }^{2}$ Maria Pia Fantini, ${ }^{3}$ David Christiani, 'Stefania Curti, ${ }^{1}$ Francesco Violante. ${ }^{1}$ Department of Medical and Surgical Sciences, University of Bologna, Bologna, Italy; ${ }^{2}$ Department of Biomedical and Neuromotor Sciences, University of Bologna, Bologna, Italy; ${ }^{3}$ School of Public Health, Harvard University, Harvard, USA

\subsection{6/oemed-2014-102362.363}

Objectives Several optimised search strategies have been developed in Medicine, and more recently in Occupational Medicine. Aim of this study is to identify efficient PubMed search strategies for the study of putative environmental determinants of a disease.

Method We compiled a list of search terms (either Medical Subjects Heading $[\mathrm{MeSH}]$ or non-MeSH) seeming pertinent to exposure to pollutants as determinants of diseases in general population. We estimated proportions of potentially pertinent articles to formulate two search strategies (one "more specific", one "more sensitive"). We applied these strategies to retrieve information on the putative environmental determinants of three diseases: autoimmune disease, sudden death and congenital heart defects. We evaluated the number of needed to read (NNR) abstracts to identify one potentially pertinent article in the context of these pathologies.

Results The "more specific" search string was based on the combination of terms that yielded the highest proportion $(\geq$ $40 \%$ ) of potentially pertinent abstracts, including the $\mathrm{MeSH}$ terms 'air pollutants', 'air pollution', 'disorders of environmental origin', 'environmental exposure' and 'particulate matter'. The "more sensitive" string was based on use of broader search fields and additional coverage provided by other search terms under study. Using the "more specific" string, the NNR to find one potentially pertinent article were: 2.7 for autoimmune disease; 3.2 for sudden death; 1.1 for congenital heart defects. Using the sensitive strategy, the NNR were 4.0, 6.1 and 3.4, respectively.

Conclusions The proposed strings could help health care professionals investigate environmental determinants of medical conditions that could be related to pollution. 